Pacific Journal of Mathematics

SEPARABLE ALGEBRAS OVER A COMMUTATIVE BANACH 


\title{
SEPARABLE ALGEBRAS OVER A COMMUTATIVE BANACH ALGEBRA
}

\author{
IAN CRAW AND SUSAN RosS
}

\begin{abstract}
We show that the projective separable algebras over a commutative Banach algebra $R$ with maximal ideal space $X$ are the same as those over $C(X)$. Two consequences of this are the identification of the Brauer group of $R$ with that of $C(X)$, and the identification of the set of covering algebras of $R$ with the finitely fibred covering spaces of $X$.
\end{abstract}

This result is part of an old programme relating the algebraic structure of $R$ with the topological structure of $X$ and we start by showing how it fits into this context. An early result of this type is the Arens-Royden theorem; that the Gelfand transform induces an isomorphism $R^{-1} / \exp R$ $\rightarrow C(X)^{-1} / \exp C(X)$ from the group of invertibles in $R$, modulo the subgroup of elements which have a logarithm. In $C(X)$, this quotient group simply describes $H^{1}(X, \mathbf{Z})$, the first Čech cohomology group of $X$ with integer coefficients and so in particular the obstruction to an element in $R^{-1}$ having a logarithm is purely topological and well understood.

Similarly, the Silov idempotent theorem can be interpreted as describing $H^{0}(X, \mathbf{Z})$. More generally, topological information about $X$ can be reflected in the structure of certain modules over $R$. Let $\mathscr{P}(R)$ denote the semigroup (under $\oplus$ ) of isomorphism classes of finitely generated projective $R$-modules. Regarding $C(X)$ as an $R$-module via the Gelfand transform gives a semigroup homomorphism $P \leadsto P \otimes_{R} C(X)$ from $\mathscr{P}(R)$ to $\mathscr{P}(C(X))$. In [13] Novodvorskii shows that this is an isomorphism and hence induces an isomorphism $K_{0}(R) \simeq K_{0}(C(X))$ between the corresponding Grothendieck groups; in particular this gives a description $K_{0}(R) \simeq K^{0}(X)$ of the topological $K$-theory of $X$ in terms of $R$. Restricting to the invertible modules gives a result of Forster [8] describing the Picard group of $R$

$$
\operatorname{Pic} R \simeq \operatorname{Pic} C(X) \simeq H^{2}(X, \mathbf{Z}) \text {. }
$$

These results, and others along the same lines can be obtained in a unified way using the fact that the corresponding topological functors have classifying spaces which are 'nice' and in particular finitely generated. A description of this method, the Novodvorskii-Taylor theorem and further background is given by Taylor in [18]; we go into detail about this in $\S 2$.

It seems significantly harder to get analogues of these results for the higher cohomology groups, and in particular for $H^{3}(X, \mathbf{Z})$, where it is 
known that a classifying space cannot be finitely generated. A result of Serre (cf. [10,1.6]) identifies the torsion subgroup of $H^{3}(X, \mathbf{Z})$ with $\operatorname{Br} C(X)$, the Brauer group of $C(X)$, and this suggests that some sort of 'infinite dimensional' Brauer group may give the whole of $H^{3}(X, \mathbf{Z})$. In [19] Taylor develops a different technique which gives results in this situation. However the theorem is not entirely satisfactory in that the algebraic objects involved are hard to recognise. A subsequent approach is described in [20].

Restricting to the torsion subgroup of $H^{3}(X, \mathbf{Z})$ should bypass a lot of these difficulties. Taylor notes this, and in [19, Theorem 6] he gives the isomorphism

$$
\operatorname{Br}(R) \simeq \operatorname{Br} C(X) \simeq \operatorname{Tor} H^{3}(X, \mathbf{Z}) .
$$

In this paper we give what we hope is a complete proof of this result. (In [19] some proofs are outlined, but otherwise details are omitted.) Our method follows Taylor's original paper [18], and we obtain rather more information by getting the isomorphism at the semigroup level.

This additional information enables us to give a solution to a slightly different problem first raised by Magid [12] and recently solved by Zame [21]. It is possible to define the notion of a covering $R$-algebra in such a way that $C(Y)$ is a covering $C(X)$-algebra precisely when $Y$ is a finitely fibred covering space of the compact Hausdorff space $X$ [2]. Such spaces are of interest topologically in that, at least when $X$ is locally well behaved, they are determined precisely by those subgroups of the fundamental group of $X$ which have finite index. In [12] Magid considered this idea in the context of commutative unital Banach algebras. He showed that if $A$ is a covering $R$ algebra, then the maximal ideal space of $A$ is indeed a finitely fibred covering space of $X$, and asked whether the converse was true; i.e. whether given $R$ and a finitely fibred covering space $Y$ of $X$, it is possible to find a covering $R$-algebra $A$ with maximal ideal space $Y$. As evidence for this conjecture Magid showed it was true when $R$ was semisimple and regular [12, Theorem 11], while in [4] a solution was given for any $R$ whose maximal ideal space was path connected and had abelian fundamental group. Finally Zame [21] showed it was always possible to find a suitable $A$ by developing what seems to be the correct notion of Galois theory in the context of commutative Banach algebras. Our solution is rather different from that given by Zame, and is obtained by specialising our main result to the commutative case.

The idea common to the two results described above is that of a separable algebra. In order to describe our main theorem precisely we first 
review the algebraic theory of these algebras. More details can be found in the books by DeMeyer and Ingraham [6] and Orzech and Small [14].

Let $R$ be a commutative ring and $A$ a unital $R$-algebra. Write $A^{0}$ for the $R$-module $A$ with the opposite multiplication to that on $A$ and let $A^{e}=A \otimes_{R} A^{0}$. Each of $A^{e}$ and $A$ is a left $A^{e}$-module in a natural way; we say that $A$ is a separable $R$-algebra if the multiplication map $m: A^{e} \rightarrow A$ has a right inverse as an $A^{e}$-module homomorphism. Equivalently, there is an element $e=\sum_{i=1}^{n} x_{i} \otimes y_{i}$ in $A \otimes_{R} A$ with $\sum x_{i} y_{i}=1$ and $\sum a x_{i} \otimes y_{i}=\sum x_{i}$ $\otimes y_{i} a(a \in A)$. Such an $e$, necessarily idempotent in $A^{e}$, is called a separability idempotent for $A$. If $A$ is both separable and projective, a result of Villamayor and Zelinsky [6, II-2-1] ensures that $A$ is finitely generated as an $R$-module. We write $\operatorname{Sep}(R)$ for the set of isomorphism classes of projective separable $R$-algebras. If $A$ and $B$ are both separable so is $A \otimes_{R} B$, whence $\operatorname{Sep}(R)$ becomes a semigroup under $\otimes_{R}$. If $\varphi: R \rightarrow S$ is a homomorphism of rings and $S$ becomes an $R$-module via $\varphi$, then $A \otimes_{R} S$ is a separable $S$-algebra whenever $A$ is a separable $R$-algebra. Thus $\varphi$ induces a semigroup homomorphism $\operatorname{Sep}(R) \rightarrow \operatorname{Sep}(S)$.

In particular if $m$ is any maximal ideal in $R$, the natural projection $R \rightarrow R / m$ induces a map $\operatorname{Sep}(R) \rightarrow \operatorname{Sep}(R / m)$. A rather deep result that we shall need enables us conversely to recognise separability locally. Note that there is a natural isomorphism $A / m A \simeq A \otimes_{R} R / m$; the following result is then given in [6, II-7.1].

Proposition 1.1. Let $A$ be a finitely generated projective $R$-algebra. Then $A \in \operatorname{Sep}(R)$ iff $A \otimes_{R} R / m \in \operatorname{Sep}(R / m)$ for every maximal ideal $m$ of $R$.

The theory of separable algebras naturally divides into two branches, since if $A$ is separable over $R$ it is separable over its centre $Z(A)$ which in turn is separable and commutative over $R$. The study of commutative separable $R$-algebras (which are faithful and projective as $R$-modules) is a generalisation of the Galois theory of fields - whence the name; it is this aspect which is studied in the context of Banach algebras by Zame [21] and these are the covering $R$-algebras noted above. The set of such $R$-algebras, $\operatorname{Cov}(R)$ is a semigroup of $\operatorname{Sep}(R)$.

The other branch of the theory is the study of central separable or Azumaya $R$-algebras, and such algebras form a subsemigroup $\operatorname{Az}(R)$ of $\operatorname{Sep}(R)$. The prototype Azumaya $R$-algebra is $M_{n}(R)$, the algebra of $n \times n$ matrices with entries from $R$, while the Azumaya $C(X)$-algebras may be 
described either as the matrix-algebra bundles on $X$, or equivalently, as the $n$-homogeneous $C^{*}$-algebras.

One way to produce Azumaya algebras generalises $M_{n}(R)=$ End $_{R}\left(R^{n}\right)$; one simply takes $\operatorname{End}_{R}(P)$ for any finitely generated faithful projective $R$-module $P$. Indeed these occur frequently since if $A \in \mathrm{Az}(R)$ $A \otimes_{R} A^{0} \simeq \operatorname{End}_{R}(A)$. Now define an equivalence relation on $\operatorname{Az}(R)$ by $A \sim B$ iff there are faithfully projective modules $P$ and $Q$ such that

$$
A \otimes_{R} \operatorname{End}_{R}(P) \simeq B \otimes_{R} \operatorname{End}_{R}(Q) .
$$

The set of such equivalence classes is the Brauer group $\operatorname{Br}(R)$, a group since by the relation noted above, $[A]^{-1}=\left[A^{0}\right]$. More conceptually, the relation $[A]=[B]$ in $\operatorname{Br}(R)$ is the statement that the categories of left $A$-modules and left $B$-modules are equivalent.

The main result of this paper, Theorem 6.5 , is that for a commutative unital Banach algebra $R$ with maximal ideal space $X$, the map $A \leadsto$ $A \otimes_{R} c(X)$ is a semigroup isomorphism $\operatorname{Sep}(R) \rightarrow \operatorname{Sep} C(X)$. It is then routine to show that this specialises to isomorphisms $\mathrm{Az}(R) \rightarrow \mathrm{Az} C(X)$ and $\operatorname{Cov}(R) \rightarrow \operatorname{Cov} C(X)$, whence we obtain the result described above. Our method of proof relies heavily on the Novodvorskii-Taylor theorem. This is outlined in the next section, at the end of which we describe the organisation of the rest of the paper.

This work originated in an M. Sc. thesis written by the second author at the University of Aberdeen in 1977-78. She gratefully acknowledges the financial support of the Fullerton, Moir and Gray Scholarship Fund during this period.

2. The Novodvorskii-Taylor theorem. We first briefly describe the holomorphic functional calculus theorem in the form we use. Let $R$ be a commutative unital Banach algebra with maximal ideal space $X$, let $i: R \rightarrow R^{* *}$ be the injection of $R$ into its bidual, and write $i(r)=\tilde{r}$ $(r \in R)$. This gives a map $R \rightarrow \vartheta(X)$, the algebra of germs of functions each holomorphic in some neighbourhood of $X \subseteq R^{*}$. For us, the functional calculus map is the unique continuous algebra homomorphism $\nu$ : $\theta(X) \rightarrow R$ which is a left inverse for $i$. This viewpoint is described in [4] or [18]; it is related to the usual statement of the theorem by the equation

$$
f\left(r_{1}, \ldots, r_{n}\right)=\nu(f \circ \tilde{\alpha})
$$

where $\alpha=\left(r_{1}, \ldots, r_{n}\right)$ and $f$ is holomorphic on a neighbourhood of sp $\alpha=$ $\{\tilde{\boldsymbol{\alpha}}(x): x \in X\} \subseteq \mathbf{C}^{n}$. 
More generally, it is convenient to deal with vector-valued functions. Let $E$ be a finite dimensional vector space. Then $\nu$ has a natural extension to a map $S: \theta(X, E) \rightarrow R \otimes E$ defined by $S=(\nu \otimes 1) \circ \psi$ where $\psi: \vartheta(X, E) \rightarrow \vartheta(X) \otimes E$ is the obvious isomorphism. If $\bar{\alpha} \in R \otimes E$ and we write $\tilde{\alpha}=\psi^{-1}(i \otimes 1) \bar{\alpha}$ then $S(\tilde{\alpha})=\bar{\alpha}$ and $S$ is an epimorphism.

Let $U, V$ be open subsets of finite dimensional spaces $E, F$ respectively and let $f: U \rightarrow V$. As in $[19, \S 1]$ write $R_{U}=\{\bar{\alpha} \in R \otimes E: \tilde{\alpha} \in$ $\theta(X, U)\}$, and define $R_{f}: R_{U} \rightarrow R_{V}$ by $R_{f}(\bar{\alpha})=S(f \circ \tilde{\alpha})$. Alternatively we may use $f$ to induce a map $\mathcal{\theta}(X)_{f}: \mathcal{\theta}(X, U) \rightarrow \mathcal{\theta}(X, V)$ by $g \leadsto f \circ g$.

Proposition 2.1. The diagram

$\begin{array}{ccc}\vartheta(X, U) & \stackrel{\theta(X)_{f}}{\rightarrow} & \vartheta(X, V) \\ \downarrow S & & \downarrow S \\ R_{U} & \stackrel{R_{f}}{\rightarrow} & R_{V}\end{array}$

commutes.

Proof. This is just a restatement of the usual composition law [1, I. 4.7]. An explicit proof in the only case we use is available in [5].

We are now in a position to describe the ingredients of the Novodvorskii-Taylor theorem. Let $X$ and $M$ be complex manifolds and let $[X, M]_{a}$ and $[X, M]$ denote the holomorphic homotopy classes of holomorphic maps, and the homotopy classes of continuous maps respectively. The basis of the theorem is a striking version of the Oka principle; for suitable $X, M$ there is a bijection

$$
[X, M]_{a} \rightarrow[X, M] .
$$

If $X$ is a Stein manifold and $M$ a Lie group this is the statement of a deep theorem of Grauert [9]. By considering complex homogeneous bundles, Ramspott [17] was able in particular to show that the theorem held when $M$ was a discrete union of complex homogeneous spaces. A further improvement was given by Taylor $[18,2.6]$; using the Arens-Calderon Lemma he showed that with the notion of holomorphicity described above, the theorem remained true when $X$ was the maximal ideal space of a Banach algebra.

With this formulation, the next step in the theorem is fairly obvious. Using the holomorphic functional calculus one passes from holomorphic 
functions to objects associated with the underlying Banach algebra $R$ to obtain

THEOREM 2.2. [18,2.7] Let $M$ be a submanifold of a domain in $\mathbf{C}^{n}$ and a discrete union of complex homogeneous spaces. Define $R_{M}=$ $\left\{\bar{\alpha} \in R^{n}: \bar{\alpha}=S(g)\right.$ for some $\left.g \in \mathcal{O}(X, M)\right\}$. Then $R_{M}$ is locally path connected and the map

$$
\left[R_{M}\right] \rightarrow[X, M]
$$

induced by the Gelfand transform on the components of $R_{M}$ is bijective.

To obtain concrete results from this theorem, one chooses an appropriate $M$ and interprets the resulting $\left[R_{M}\right]$. The arguments here are usually special to the particular $M$; however Taylor gave an alternative description of $R_{M}$ which will assist us. Let $\theta_{z}$ denote the ring of germs of functions each holomorphic in some neighborhood of $z$, and let $I(M)_{z}$ be the ideal in $\theta_{z}$ consisting of germs of functions which vanish on $M$.

Proposition 2.3. Let $M$ be a closed submanifold of an open subset $U \subseteq \mathbf{C}^{n}$, and let $F$ be a subset of $\Theta(U)$ which generates $I(M)_{z}$ for each $z \in U$. Then

$$
R_{M}=\left\{\bar{\alpha} \in R^{n}: \operatorname{sp}(\bar{\alpha}) \subseteq U \text { and } g(\bar{\alpha})=0 \text { for all } g \in F\right\} .
$$

We now outline the organisation of the remainder of the paper. Working over an arbitrary ground ring $R$, we define in $\S 3$ a set $S_{k}(R)$ which describes certain projective separable $R$-algebras, and do the necessary algebraic bookkeeping. In $\S 4$ we specialise to the case when $R$ is Banach and draw topological consequences, the main one being that in some sense $S_{k}(R)$ is stable under small perturbations. The set $S_{k}(\mathbf{C})=S_{k}$ is the manifold $M$ to which we apply the Novodvorskii-Taylor theorem. In $\$ 5$ we do the differential geometry needed to show that these results are applicable, while in $\S 6$ we show that $S_{k}(R)$ is a concrete realisation of $R_{S_{k}}$ and hence interpret the conclusion of the theorem.

3. Some algebraic results. Let $R$ be a commutative ring. We shall describe unital $R$-algebras which are finitely generated and projective as $R$-modules in terms of a projection $p: R^{k} \rightarrow R^{k}$, a multiplication $m$ on $p\left(R^{k}\right)$ and an identity $i \in p\left(R^{k}\right)$.

For positive integers $n, k$ let $L^{n}\left(R^{k}\right)$ be the $R$-module of maps $R^{k} \times \cdots \times R^{k} \rightarrow R^{k}$ which are $R$-linear in each variable, and conventionally write $L^{0}\left(R^{k}\right)$ for $R^{k}$. Let $E_{k}(R)=L^{1}\left(R^{k}\right) \oplus L^{2}\left(R^{k}\right) \oplus L^{0}\left(R^{k}\right)$ and 
define maps $f_{1}, f_{5}, f_{6}: E_{k}(R) \rightarrow L^{1}\left(R^{k}\right), f_{2}: E_{k}(R) \rightarrow L^{2}\left(R^{k}\right), f_{3}: E_{k}(R) \rightarrow$ $L^{3}\left(R^{k}\right)$ and $f_{4}: E_{k}(R) \rightarrow L^{0}\left(R^{k}\right)$ as follows:

$$
\begin{aligned}
& f_{1}(p, m, i)=x \rightsquigarrow p(p(x))-p(x) \\
& f_{2}(p, m, i)=(x, y) \rightsquigarrow p m(p x, p y)-m(x, y) \\
& f_{3}(p, m, i)=(x, y, z) \rightsquigarrow m(x, m(y, z))-m(m(x, y), z) \\
& f_{4}(p, m, i)=i-p i \\
& f_{5}(p, m, i)=x \rightsquigarrow m(x, i)-p x \\
& f_{6}(p, m, i)=x \rightsquigarrow m(i, x)-p x,
\end{aligned}
$$

where $(p, m, i) \in E_{k}(R)$ and $x, y, z \in R^{k}$ are arbitrary. Thus, writing $f=\left(f_{1}, \cdots, f_{6}\right)$ we have

$$
\begin{aligned}
f: E_{k}(R) \rightarrow & F_{k}(R)=L^{1}\left(R^{k}\right) \oplus L^{2}\left(R^{k}\right) \oplus L^{3}\left(R^{k}\right) \\
& \oplus L^{0}\left(R^{k}\right) \oplus L^{1}\left(R^{k}\right) \oplus L^{1}\left(R^{k}\right) .
\end{aligned}
$$

We write $f=f_{R}$ when it is necessary to specify the underlying ring.

Let $N_{k}(R)=\left\{\alpha \in E_{k}(R): f(\alpha)=0\right.$ in $\left.F_{k}(R)\right\}$, and fix $\alpha=(p, m, i)$ $\in N_{k}(R)$. Since $f_{1}(\alpha)=0, p$ is an $R$-linear projection on $R^{k}$, and hence $p\left(R^{k}\right)$ is a finitely generated projective $R$-module. Since $f_{3}(\alpha)=0, m$ is an associative multiplication under which $R^{k}$ is an $R$-algebra; this restricts to an $R$-algebra structure on $p\left(R^{k}\right)$ since $f_{2}(\alpha)=0$. We also have $f_{4}(\alpha)=$ $f_{5}(\alpha)=f_{6}(\alpha)=0$ so $i \in p\left(R^{k}\right)$ and acts as a 2-sided identity for the multiplication on $p\left(R^{k}\right)$. We write $\alpha\left(R^{k}\right)$ for the module $p\left(R^{k}\right)$ equipped with this structure as a unital $R$-algebra. It thus makes sense to define

$$
S_{k}(R)=\left\{\alpha \in N_{k}(R): \alpha\left(R^{k}\right) \text { is a separable } R \text {-algebra }\right\} \text {. }
$$

Conversely suppose $A$ is a projective separable unital $R$-algebra. Since $A$ is necessarily finitely generated there is a positive integer $k$ and an $R$-linear projection $p$ such that $A \simeq p\left(R^{k}\right)$ an $R$-module. By extending the multiplication to be zero off $p\left(R^{k}\right)$, we thus obtain $\alpha \in S_{k}(R)$ with $A \simeq \alpha\left(R^{k}\right)$. However, note that neither $\alpha$, nor indeed $k$ is determined uniquely by $A$. Given $\alpha \in S_{k}(R)$ we denote by $\alpha \oplus 0$ the element of $S_{k+l}(R)$ obtained in the obvious way from the embedding of $R^{k}$ in $R^{k} \oplus R^{l}$. Clearly $(\alpha \oplus 0)\left(R^{k+l}\right) \simeq \alpha\left(R^{k}\right)$.

To keep track of the ambiguity involved in the choice of $\alpha$, define an action of $G L_{k}(R)$, the group of invertible elements of $L^{1}\left(R^{k}\right)$, on $L^{n}\left(R^{k}\right)$ by

$$
u \cdot T=\left(x_{1}, \ldots, x_{n}\right) \leadsto u T\left(u^{-1} x_{1}, \ldots, u^{-1} x_{n}\right)
$$


where $u \in G L_{k}(R), T \in L^{n}\left(R^{k}\right), x_{1}, \ldots, x_{n} \in R^{k}$, and $n>0$, and on $L^{0}\left(R^{k}\right)$ by $u \cdot x=u(x)$. By acting coordinatewise, this defines actions of $G L_{k}(R)$ on each of $E_{k}(R)$ and $F_{k}(R)$.

LEMMA 3.1. The set $N_{k}(R)$ is invariant under the action of $G L_{k}(R)$. If $\alpha \in N_{k}(R)$ and $\beta=u \cdot \alpha$ then $\beta\left(R^{k}\right) \simeq \alpha\left(R^{k}\right)$, whence $S_{k}(R)$ is also invariant under the action of $G L_{k}(R)$.

Proof.

$$
\begin{aligned}
u \cdot f_{1}(\alpha)(x) & =u p^{2} u^{-1} x-u p u^{-1} x \\
& =u p u^{-1}\left(u p u^{-1} x-x\right) \\
& =f_{1}(u \cdot \alpha)(x), \text { where } \alpha=(p, m, i) \text { and } x \in R^{k}
\end{aligned}
$$

Five similar computations show that $u \cdot f(\alpha)=f(u \cdot \alpha)$, and in particular if $f(\alpha)=0$ then $f(u \cdot \alpha)=0$. The remainder of the lemma now follows, since if $\beta=u \cdot \alpha, u: \alpha\left(R^{k}\right) \rightarrow \beta\left(R^{k}\right)$ is an isomorphism of $R$-algebras.

We next investigate the way these sets behave under a change of rings. If $\varphi: R \rightarrow S$ is a ring homomorphism it induces maps from $L^{n}\left(R^{k}\right)$ to $L^{n}\left(S^{k}\right)$ and hence also from $E_{k}(R)$ to $E_{k}(S)$ and from $F_{k}(R)$ to $F_{k}(S)$. We use $\varphi_{*}$ for each of these maps. For example if $m \in L^{2}\left(R^{k}\right)$ is given using the canonical basis in $R^{k}$ by $m\left(e_{l}, e_{j}\right)=\sum r_{i j l} e_{l}$, then $\varphi_{*}(m)$ is the $S$-bilinear map

$$
\left(\sum s_{l} e_{l}, \sum s_{j} e_{J}\right) \rightsquigarrow \sum s_{i} s_{J} \varphi_{*}\left(r_{l j l}\right) e_{l} .
$$

It is of course perfectly possible to describe the above situation functorially, but it seems appropriate to resist the temptation. Note however that the assignment $\varphi \leadsto \varphi_{*}$ is clearly covariant.

LEMMA 3.2. Let $\varphi: R \rightarrow S$ be a ring homomorphism and induce maps $\varphi_{*}$ as above. Then the diagram

$$
\begin{array}{ccc}
E_{k}(R) & \stackrel{f_{R}}{\rightarrow} & F_{k}(R) \\
\downarrow \varphi_{*} & & \downarrow \varphi_{*} \\
E_{k}(S) & \stackrel{f_{S}}{\rightarrow} & F_{k}(S)
\end{array}
$$

commutes. In particular, $\varphi_{*}$ maps $N_{k}(R)$ into $N_{k}(S)$. 
Proof. Write $p=\Sigma r_{i j} e_{i j}$ where $\left\{e_{i j}\right\}$ is the usual basis in $L^{1}\left(R^{k}\right)$. Then

$$
\begin{aligned}
\varphi_{*} f_{1}(p) & =\varphi_{*}\left(\sum r_{i j} r_{j k} e_{i k}-\sum r_{i k} e_{i k}\right) \\
& =\sum\left(\varphi\left(r_{i j}\right) \varphi\left(r_{j k}\right)-\varphi\left(r_{i k}\right)\right) e_{i k}=f_{1} \varphi_{*}(p) .
\end{aligned}
$$

Five similar computations establish the commutativity of the diagram. If $f_{R}(\alpha)=0$, then $f_{S}\left(\varphi_{*}(\alpha)\right)=0$ and so $\varphi_{*}(\alpha) \in N_{k}(S)$.

It seems necessary to prove the above result in order to keep track of a particular embedding of $A \in \operatorname{Sep}(R)$ in $R^{k}$. Recall from $\S 1$ that if $\varphi$ : $R \rightarrow S$ and we regard $S$ as an $R$-algebra via $\varphi$, then $A \otimes_{R} S \in \operatorname{Sep}(S)$. The following result shows there is no confusion between these two ways of changing rings.

LEMMA 3.3. Let $\alpha \in N_{k}(R)$ and let $A=\alpha\left(R^{k}\right)$. The isomorphism $R^{k} \otimes_{R} S \simeq S^{k}$ induces an isomorphism

$$
A \otimes_{R} S \simeq \varphi_{*}(\alpha)\left(S^{k}\right)
$$

of $S$ algebras. In particular $\varphi_{*}$ maps $S_{k}(R)$ into $S_{k}(S)$.

Proof. Let $\alpha=(p, m, i)$. Tensoring the decomposition $p\left(R^{k}\right) \oplus$ $(1-p)\left(R^{k}\right)$ with $S$ preserves the direct sum, and under the isomorphism $R^{k} \otimes_{R} S \simeq S^{k}$ it is easy to check that the induced projection is just $\varphi_{*}(p)$. This gives a commutative diagram

$$
\begin{array}{ccc}
R^{k} \otimes_{R} S & \rightarrow & S^{k} \\
\downarrow p \otimes 1 & & \downarrow \varphi_{*}(p) \\
p\left(R^{k}\right) \otimes S & \rightarrow & \varphi_{*}(p)\left(S^{k}\right)
\end{array}
$$

in which the horizontal arrows are isomorphism. It is now elementary to check that the lower arrow is an $S$-algebra isomorphism when $p\left(R^{k}\right)$ is given the unital algebra structure induced by $\alpha$. Using this and the fact that $A \in \operatorname{Sep}(R) \Rightarrow A \otimes_{R} S \in \operatorname{Sep}(S)$ gives the last part of the lemma.

4. The perturbation theorem. We now specialise to the case in which $R$ is a commutative unital Banach algebra. Topologise $R^{k}$ with the $l^{\infty}$-norm. Then if $T: R^{k} \times \cdots \times R^{k} \rightarrow R^{k}$ is $R$-linear in each variable, it is necessarily continuous, and $L^{n}\left(R^{k}\right)$ becomes a Banach space when given the usual operator norm. Again using the $l^{\infty}$-norm we thus norm each of $E_{k}(R)$ and $F_{k}(R)$; in particular $N_{k}(R)$ and $S_{k}(R)$ inherit topologies as subspaces of $E_{k}(R)$. We shall make essential use of the following version of the Johnson-Raeburn-Taylor perturbation theorem [11], [16]. 
THEOREM 4.1. Let $\alpha \in S_{k}(R)$. There are constants $\delta>0$ and $C>0$ such that given $\beta \in N_{k}(R)$ with $\|\alpha-\beta\|<\delta<1$ there is some $u \in$ $G L_{k}(R)$ with $\|u-1\|<C\|\alpha-\beta\|$ such that $\beta=u \cdot \alpha$.

Proof. We first obtain two multiplications on the same underlying space. Let $\alpha=(p, m, i), \beta=(q, n, j)$ and define $v: p\left(R^{k}\right) \rightarrow q\left(R^{k}\right)$ by

$$
v=q p+(1-q)(1-p) \text {. }
$$

Then $v-1=(q-p)(2 p-1)$ and so provided $\delta<\|2 p-1\|^{-1}$ we have $v \in G L_{k}(R)$ and $q=v \cdot p$. There is then a constant $K_{1}$ depending only on $\alpha$, such that

$$
\max \left(\|v-1\|,\left\|v^{-1}-1\right\|\right)<K_{1}\|\alpha-\beta\| .
$$

Let $\gamma=v \cdot \alpha$ so by Lemma $3.1 \gamma \in S_{k}(R)$, and

$$
\begin{aligned}
\|\gamma-\alpha\| & \leq \max (\|p-q\|,\|m-v \cdot m\|,\|1-v\|\|i\|) \\
& \leq\|\alpha-\beta\| \max \left(1,\|\alpha\|\left(3 K_{1}+2 K_{1}^{2}\right),\|\alpha\| K_{1}\right) \\
& \leq K_{2}\|\alpha-\beta\| \text { where } K_{2}=K_{2}(\alpha) .
\end{aligned}
$$

Thus $\|\beta-\gamma\| \leq\left(1+K_{2}\right)\|\alpha-\beta\|$, and for small $\delta$ we have in particular two close multiplications on $q\left(R^{k}\right)$.

Let $A=\gamma\left(R^{k}\right)$. Since $A$ is separable, with splitting idempotent $\sum x_{i} \otimes y_{i}$ say, it follows that if $\delta: A \rightarrow M$ is any $R$-linear derivation into an $R$-module $M$, then $\delta$ is inner being implemented by $\sum x_{i} \delta\left(y_{i}\right)$. [14,4.11; this is a standard characterisation of separability]. Thus for the purely algebraic Hochschild cohomology over $R$ we have $H_{R}^{2}(A, A)=H_{R}^{3}(A, A)$ $=0$.

Further since $A=q\left(R^{k}\right)$ and $q$ is continuous, $A$ is a closed submodule of $R^{k}$, and hence itself a Banach space. Further the multiplication $\pi=v \cdot m$ is continuous and hence $A$ can be renormed with an equivalent norm under which it is a Banach algebra. In this norm we have $\|n-\pi\|$ $<K_{3}\|\alpha-\beta\|$. We are thus almost in a position to apply the perturbation theorem [11, Theorem 2.1], [16, Theorem 3] to obtain an invertible map $w$ from $A$ to $A$ with $\| w-$ id $\left\|<K_{4}\right\| n-\pi \|$ such that

$$
n(x, y)=w \pi\left(w^{-1} x, w^{-1} y\right) \quad(x, y \in A) .
$$

Indeed all of our $R$-linear maps are continuous, and an examination of the proof of the perturbation theorem shows that in our case the derivatives of the appropriate maps will be $R$-linear and hence maps for which we can apply the vanishing of $H_{R}^{2}(A, A)$. The only point then in doubt is that $w$ : $A \rightarrow A$ is $R$-linear, and this can easily be checked from the construction given in [11, Theorem 2.1]. 
Since $w: q\left(R^{k}\right) \rightarrow q\left(R^{k}\right)$ we may define $\tilde{w} \in G L_{k}(R)$ by

$$
\tilde{w}(x)=w q(x)+(1-q)(x) \quad\left(x \in R^{k}\right) .
$$

Then $q \tilde{w}=\tilde{w} q$, and

$$
\|\tilde{w}-1\| \leq\|w-\mathrm{id}\|\|q\| \leq K_{5}\|\alpha-\beta\|
$$

where we now work again with the original norm on $A$. We have $\beta=(w \circ v) \cdot \alpha$ and

$$
\begin{aligned}
\|\tilde{w} \circ v-1\| & \leq\left(\|v\| K_{5}+K_{1}\right)\|\alpha-\beta\| \\
& \leq C\|\alpha-\beta\|
\end{aligned}
$$

where again since $\delta<1, C$ depends only on $\alpha$.

This result puts us in a position to describe the ambiguity involved in associating $\alpha \in S_{k}(R)$ with a separable algebra $A$. Note first that an application of Theorem 4.1 together with the fact that the connected component of the identity in $G L_{k}(R)$ consists of (finite products of) exponentials shows that as a topological space, $S_{k}(R)$ is locally path connected. We write $\alpha \sim \beta$ iff $\alpha$ and $\beta$ are in the same component of $S_{k}(R)$, and the set of path components as $\left[S_{k}(R)\right]$.

Proposition 4.2. Let $R$ be a commutative unital Banach algebra. Let $\alpha_{0}, \alpha_{1} \in S_{k}(R)$ and assume $\alpha_{0} \sim \alpha_{1}$. Then $\alpha_{0}\left(R^{k}\right) \simeq \alpha_{1}\left(R^{k}\right)$. Conversely if $\alpha, \beta \in S_{k}(R)$ and $\alpha\left(R^{k}\right) \simeq \beta\left(R^{k}\right)$ then $\alpha \oplus 0 \sim \beta \oplus 0$ in $S_{4 k}(R)$.

Proof. Let $\alpha_{t}(0 \leq t \leq 1)$ be the path in $S_{k}(R)$ connecting $\alpha_{0}$ and $\alpha_{1}$. Let

$$
\tau=\sup \left\{t: \alpha_{t}\left(R^{k}\right) \simeq \alpha_{0}\left(R^{k}\right)\right\}
$$

Then $\alpha_{\tau} \in S_{k}(R)$, and by Theorem 4.1 and Lemma 3.1 there is a neighbourhood $U$ of $\alpha_{\tau}$ in $N_{k}(R)$ such that if $\beta \in U$ then $\beta\left(R^{k}\right) \simeq \alpha_{\tau}\left(R^{k}\right)$. Thus $\tau=1$ and $\alpha_{1}\left(R^{k}\right) \simeq \alpha_{0}\left(R^{k}\right)$.

The converse is almost the same as the corresponding result for finitely generated projective modules [17,6.7]. Let $u: \alpha\left(R^{k}\right) \rightarrow \beta\left(R^{k}\right)$ be the given isomorphism, and define an isomorphism on $R^{2 k}$, say

$$
\begin{aligned}
v: p R^{k} \oplus(1-p) R^{k} \oplus q\left(R^{k}\right) \oplus(1-q) R^{k} \rightarrow & q R^{k} \oplus(1-q) R^{k} \\
& \oplus p R^{k} \oplus(1-p) R^{k}
\end{aligned}
$$


by $v(a, b, c, d)=\left(u(a), d, u^{-1}(c), b\right)$, where $\alpha=(p, m, i)$ and $\beta=$ $(q, n, j)$. Then $\beta \oplus 0=v \cdot(\alpha \oplus 0)$. Let $w=v \oplus v^{-1}: R^{4 k} \rightarrow R^{4 k}$, so $w \in$ $G L_{4 k}(R)$ and $\beta \oplus 0=w \cdot(\alpha \oplus 0)$ in $S_{4 k}(R)$. Furthermore

$$
t \leadsto w_{t}=\frac{1}{2}\left(\begin{array}{cc}
(1+v)+(1-v) \cos t & (1-v) \sin t \\
\left(v^{-1}-1\right) \sin t & \left(1+v^{-1}\right)+\left(1-v^{-1}\right) \cos t
\end{array}\right)
$$

is a path in $G L_{4 k}(R)$ with $w_{0}=1, w_{\pi}=w$. Thus $\alpha \oplus 0 \sim \beta \oplus 0$.

5. Differential geometry. We now specialise to the case $R=\mathbf{C}$ and abbreviate $E_{k}(R)$ etc. as $E_{k}$. Our aim in this section is to obtain detailed information about the way $S_{k}$ sits inside $E_{k}$ which will allow us to apply the Novodvorskii-Taylor theorem.

The first problem is to show that each of the orbits of $G L_{k}$ acting on $S_{k}$ is a submanifold of $E_{k}$. Given this, a standard Lie group argument would show that each orbit was a complex homogeneous space. In fact it seems necessary to work the other way round; our tool is the following elementary result given by Raeburn for Banach Lie groups in [15, 1.5]. The proof for Lie groups is exactly the same, we simply obtain the complementation requirements automatically at each stage of the argument.

Proposition 5.1. Let $E$ be a finite dimensional linear space on which the Lie group $G$ acts holomorphically. For $\alpha \in E$ let $\pi^{\alpha}(u)=u \cdot \alpha$ be the map $G \rightarrow E$ induced by the action, and let $M=\pi^{\alpha}(G)$. Assume that $\pi^{\alpha}$ : $G \rightarrow M$ is an open map. Then $M$ is a complex homogeneous space and $a$ submanifold of $E$.

Proposition 5.2. The set $S_{k}$ is a discrete union of homogeneous spaces. Further, there is an open neighbourhood $U$ of $S_{k}$ in $E_{k}$ such that $U \cap N_{k}=S_{k}$.

Proof. Pick $\alpha \in S_{k}$ and note first that if the group action $G L_{k} \times E_{k} \rightarrow$ $E_{k}$ is that described in $\S 3$, then $\pi^{\alpha}\left(G L_{k}\right) \subseteq S_{k}$ by Lemma 3.1. We can apply the perturbation theorem (Theorem 4.1) to obtain constants $C, \delta>0$ such that

$$
\pi^{\alpha}\left\{u \in G L_{k}:\|u-1\|<C \delta\right\} \subseteq\left\{\beta \in S_{k}:\|\alpha-\beta\|<\delta\right\} .
$$

A translation argument then shows that $\pi^{\alpha}: G L_{k} \rightarrow S_{k}$ is an open map and so we can apply Proposition 5.1.

Let $\left\{S_{k i}: i \in I\right\}$ be the distinct orbits we obtain in this way so that

$$
S_{k}=U\left\{S_{k i}: i \in I\right\}
$$


and each $S_{k i}$ is a complex homogeneous space. Note that for each $\alpha \in S_{k i}$, the perturbation theorem shows there is an open ball $B_{\alpha}$ in $E_{k}$ centred at $\alpha$ such that $B_{\alpha} \cap N_{k} \subseteq S_{k i}$. By passing to balls of half this radius we thus obtain neighbourhoods $U_{i}$ of $S_{k i}$ with $U_{i} \cap U_{j}=\varnothing(i \neq j)$. Thus $S_{k}$ is indeed a discrete union of complex homogeneous spaces, while putting $U=U\left\{U_{i}: i \in I\right\}$ we have $U \cap N_{k}=S_{k}$.

COROLLARY 5.3. The set $S_{k}$ is a closed submanifold of an open subset of some $\mathbf{C}^{n}$.

Proof. Clearly $N_{k}$ is closed in $E_{k}$ and so the result follows from the above on choosing a basis for $E$.

REMARK 5.4. We have been unable to prove that $S_{k}$ is itself closed in $N_{k}$ (for example, by showing that each of the orbits of $G L_{k}$ in $N_{k}$ is relatively open). Indeed the action of $\frac{1}{n} \cdot 1$ on the data for the matrix algebra $M_{k}(\mathbf{C})$, whose multiplication converges to the zero multiplication, suggests the result is false. We are thus unable to apply the DocquierGrauert theorem to obtain a holomorphic retract onto $S_{k}$. Were such a retract available, the simplest course at this point would be to apply Theorem 2.2 directly, using the image of the retract under the functional calculus to appropriately identify $R_{M}$. One can in fact manufacture a retract onto a larger version of $M$, and this is the route Taylor follows in [19, Proposition 1]. However in our case this would be close to reproving the characterisation given in Proposition 2.3; we choose instead to apply this directly.

To do this, we again identify $E_{k}$ with $\mathbf{C}^{n}=\mathbf{C}^{k+k^{2}+k^{3}}$ and similarly for $F_{k}$. Restricting $f: E_{k} \rightarrow F_{k}$ to $U$ clearly gives a holomorphic map, and composing with the various coordinate projections on $F_{k}$ gives a finite set $F \subseteq \mathcal{O}(U)$ such that

$$
S_{k}=\{z \in U: g(z)=0 \text { for all } g \in F\}
$$

on using Proposition 5.2.

Consider the composition $S_{k} \stackrel{i}{\rightarrow} U \stackrel{f}{\rightarrow} F_{k}$ where $i$ is the identity map. If $\alpha \in S_{k}$ then since $f \circ i=0$ we have image $\left(d i_{\alpha}\right) \subseteq \operatorname{ker} d f_{\alpha}$. This image is simply $T_{\alpha}\left(S_{k}\right)$, the tangent space to $S_{k}$ at $\alpha$, regarded as embedded in $E_{k}$. Thus

$$
T_{\alpha}\left(S_{k}\right) \subseteq \operatorname{ker} d f_{\alpha}
$$


In fact we have equality, although since we do not yet know that the rank of $f$ is locally constant at $\alpha$ we cannot use the usual implicit function theorem argument. Instead we compute to obtain the result directly.

Proposition 5.5. $T_{\alpha}\left(S_{k}\right)=\operatorname{ker}\left(d f_{\alpha}\right)$.

Proof. We first compute $T_{\alpha}\left(S_{k}\right)$. Let $\alpha=(p, m, i)$. Since $\pi^{\alpha}$ is an open map, and a neighbourhood of the identity in $G L_{k}$ consists of exponentials, we have

$$
\begin{gathered}
T_{\alpha}\left(S_{k}\right)=\left\{\left.\frac{d}{d t}\left(e^{t u} \cdot \alpha\right)\right|_{t=0}: u \in M_{k}\right\}, \\
=\left\{(q, n, j) \in E_{k}\right\} \quad \text { say, where } \\
q=\left.\frac{d}{d t}\left(e^{t u} \cdot p\right)\right|_{t=0} ; \quad n=\left.\frac{d}{d t}\left(e^{t u} \cdot m\right)\right|_{t=0} ; \quad j=\left.\frac{d}{d t}\left(e^{t u} \cdot i\right)\right|_{t=0}
\end{gathered}
$$

Thus

$$
\begin{gathered}
q=u p-p u \\
n(x, y)=u m(x, y)-m(x, u y)-m(u x, y) \\
j=u i
\end{gathered}
$$

where $x, y \in \mathbf{C}^{k}$ are arbitrary. Now pick $\beta=(q, n, j) \in \operatorname{ker} d f_{\alpha}$. Then since $\left(d f_{i}\right)_{\alpha}(\beta)=0(1 \leq i \leq 6)$ we obtain

$$
\begin{gathered}
p q+q p-q=0 \\
n(x, y)-p n(p x, p y)-q m(x, y)-m(q x, y)-m(x, q y)=0 \\
n(x, m(y, z))+m(x, n(y, z))-n(m(x, y), z) \\
-m(n(x, y), z)=0 \\
n(x, i)+m(x, j)-q x=0 \\
n(i, x)+m(j, x)-q x=0 \\
j-p j-q i=0 .
\end{gathered}
$$

We thus have to show how these equations give $u \in M_{k}(\mathbf{C})$ such that (1) (2) and (3) hold.

Let $T: p\left(\mathbf{C}^{k}\right) \times p\left(\mathbf{C}^{k}\right) \rightarrow p\left(\mathbf{C}^{k}\right)$ be the bilinear map $(p x, p y) \leadsto$ $p n(p x, p y)$. Temporarily denoting the multiplication on $P\left(\mathbf{C}^{k}\right)$ by juxtaposition, since $x y=p x \cdot p y$ we have $x T(y, z)-T(x y, z)+T(x, y z)$ $\left.-T(x, y) z=0(x, y, z) \in p\left(\mathbf{C}^{k}\right)\right)$ on using (6). Thus $T$ is a 2-cocyle for the separable algebra $A=\alpha\left(\mathbf{C}^{k}\right)$ and hence, as in the proof of Theorem 4.1 there is some $w \in L^{\mathrm{l}}(A)$, which can be trivially extended to give 
$w \in L^{1}\left(\mathbf{C}^{k}\right)$ such that for $x, y, z \in \mathbf{C}^{k}, p n(p x, p y)=m(x, w p y)-$ $p w p m(x, y)+m(w p x, y)$. Put $u=q-2 p q-p w p$. Then

$$
u p-p u=q p+p q-2 p q p
$$

and by (4), since $p q=p q+p q p$ we have $p q p=0$. Using (4) again shows that $q=u p-p u$, and we have established (1).

From (5) and the definition of $w$ we have

$$
\begin{aligned}
n(x, y)= & (q-p w p) m(x, y)+m((q+p w p) x, y) \\
& +m(x,(q+p w p) y)
\end{aligned}
$$

and so

$$
\begin{aligned}
n(x, y)= & (u+2 p q) m(x, y) \\
& +m((2 q-u-2 p q) x, y)+m(x,(2 q-u-2 p q) y) .
\end{aligned}
$$

Since $m=p m(p \times p)$, this gives (2). Finally, using (7) together with (1) and (2) shows that $m(x, j)=m(x, u i)$ so that $p j=p u i$. Thus from (9) we see that $j=u i$ as claimed.

This calculation is a generalisation of the corresponding one for idempotents carried out in [18, Theorem 3.8] and it is used in the same way. Thus let $\theta_{z}$ denote the ring of germs of functions each holomorphic in some neighbourhood of $z$. Then using standard differential geometry [see e.g. 7, 16.8.9] we obtain the following

5.6. Corollary. $F$ generates $I\left(S_{k}\right)_{z}$, the ideal in $\theta_{z}$ of germs of functions vanishing on $S_{k}$, for each $z \in U$.

6. The main theorem. Throughout this section $R$ will again be a commutative unital Banach algebra with maximal ideal space $X$. Since the Gelfand transform $\gamma: R \rightarrow C(X)$ induces a map $\gamma_{*}: S_{k}(R) \rightarrow S_{k}(C(X))$, and since $\gamma_{*}$ is continuous with the topologies given in $\S 4$, it induces a map between components. We can now give the first version of our main result

THEOREM 6.1. The map $\gamma_{*}:\left[S_{k}(R)\right] \rightarrow\left[S_{k}(C(X))\right]$ is bijective.

Proof. By Proposition 5.2 and Corollary 5.3 we are in a position to apply the Taylor-Novodvorskii Theorem (2.3). Thus the Gelfand transform induces a bijection $\left[R_{S_{k}}\right] \rightarrow\left[X, S_{k}\right]$ and using Proposition 2.3 and Corollalry 5.6,

$$
R_{S_{k}}=\left\{\bar{\alpha} \in R^{n}: \operatorname{sp}(\bar{\alpha}) \subseteq U \text { and } g(\bar{\alpha})=0 \text { for all } g \in F\right\} .
$$

We identify the spaces involved in a series of Lemmas. 
LEMMA 6.2. There are natural isomorphisms

$$
E_{k}(C(X)) \rightarrow C\left(X, E_{k}\right) ; \quad E_{k}(\vartheta(X)) \rightarrow \vartheta\left(X, E_{k}\right) .
$$

In particular, the first of these induces a topological isomorphism $S_{k}(C(X))$ $\rightarrow C\left(X, S_{k}\right)$ and hence an identification $\left[S_{k}(C(X))\right]=\left[X, S_{k}\right]$.

Proof. Let $\varepsilon_{x}: C(X) \rightarrow \mathbf{C}$ be the evaluation map at $x \in X$. This induces a map $\left(\varepsilon_{x}\right)_{*}: E_{k}(C(X)) \rightarrow E_{k}$ which is of course continuous as a function of $x$. Thus we obtain

$$
\varepsilon: E_{k}(C(X)) \rightarrow C\left(X, E_{k}\right)
$$

and it is clear that this is bijective. The argument for $\theta(X)$ proceeds in the same way.

Restricting $\varepsilon$ to $S_{k}(C(X))$, the image certainly lies in $C\left(X, S_{k}\right)$ by Lemma 3.3. Conversely if $\alpha \in E_{k}(C(X))$ and $\varepsilon(\alpha) \in C\left(X, S_{k}\right)$, it is clear that $\alpha \in N_{k}(C(X))$ since if an equation between matrices of functions fails to hold, it will fail at some point of the underlying space. It is thus sufficient to check that $\alpha\left(C(X)^{k}\right)$ is separable. However since the quotient map modulo any maximal ideal $m$ is given by some $\varepsilon_{x}$ we have, $\alpha\left(C(X)^{k}\right) \otimes_{R} C(X) / m=\varepsilon_{x}\left(\mathbf{C}^{k}\right) \in \operatorname{Sep}(\mathbf{C})$, whence $\alpha \in \operatorname{Sep} C(X)$ by Proposition 1.1.

In order to identify $R_{S_{k}}$ we make explicit certain isomorphisms. We have already used the identification of $E_{k}$ with $\mathbf{C}^{n}$ to embed $S_{k}$ in $\mathbf{C}^{n}$. In the same way we identify $R^{n}$ with $R \otimes E_{k}$. Let $J: R \otimes E_{k} \rightarrow E_{k}(R)$ be the obvious isomorphism, and for $\bar{\alpha} \in R \otimes E_{k}$, write $J(\bar{\alpha})=\alpha$. The following description of $R_{S_{k}}$ is a consequence of the composition property for the functional calculus.

LEMMA 6.3. Let $F$ and $U$ be as described in \$5. Then for $\alpha \in E_{k}(R)$ with $\operatorname{sp}(\bar{\alpha}) \subseteq U$, we have $g(\bar{\alpha})=0$ for all $g \in F$ iff $f_{R}(\alpha)=0$.

Proof. As in $\S 2$ we write $\tilde{\alpha}$ for the image of $\alpha$ under the composition

$$
E_{k}(R) \rightarrow R \otimes E_{k} \rightarrow R^{* *} \otimes E_{k} \rightarrow \vartheta\left(X, E_{k}\right)
$$

so that $S(\tilde{\alpha})=\bar{\alpha}$. Since $\operatorname{Sp}(\bar{\alpha}) \subseteq U$, we have $\tilde{\alpha} \in \mathcal{O}(X, U)$ and again as in $\S 2, S(g \circ \tilde{\alpha})=g(\bar{\alpha})$ for any scalar-valued $g$ holomorphic on $U$. Thus $g(\bar{\alpha})=0$ for all $g \in F$ iff $S(f \circ \tilde{\alpha})=0$ where $f: U \rightarrow F_{k}$ is the map which defines $S_{k}$. 
Regarding $f$ as a map $E_{k} \rightarrow F_{k}$ gives the diagram

$$
R \otimes E_{k} \stackrel{J}{\rightarrow} E_{k}(R)
$$

$$
\begin{array}{ccc}
\downarrow R_{f} & & \downarrow f_{R} \\
R \otimes F_{k} & \stackrel{J}{\rightarrow} & F_{k}(R)
\end{array}
$$

which we claim commutes. Assuming this, and again restricting $f$ to $U$ we have

$$
\begin{array}{ccccc}
\vartheta(X, U) & \stackrel{s}{\rightarrow} & R_{U} & \stackrel{J}{\rightarrow} & E_{k}(R) \\
\downarrow \vartheta(X)_{f} & & \downarrow R_{f} & & \downarrow f_{R} \\
\vartheta\left(X, F_{k}\right) & \stackrel{s}{\rightarrow} & R \otimes F_{k} & \stackrel{J}{\rightarrow} & F_{k}(R)
\end{array}
$$

which commutes by Proposition 2.2. Thus for $\alpha \in E_{k}(R)$ with $\operatorname{sp}(\bar{\alpha}) \subseteq U$ we have

$$
f_{R}(\alpha)=J R_{f}(\bar{\alpha})=J R_{f}(S(\tilde{\alpha}))=J S(f \circ \tilde{\alpha})
$$

and the result follows since $J$ is a linear isomorphism.

To check that (1) commutes consider the diagram

$$
\begin{array}{ccccccc}
E_{k}(\vartheta(X)) & \rightarrow & \vartheta\left(X, E_{k}\right) & \stackrel{S}{\rightarrow} & R \otimes E_{k} & \stackrel{J}{\rightarrow} & E_{k}(R) \\
\downarrow f_{\Theta(X)} & & \downarrow \vartheta(X)_{f} & & \downarrow R_{f} & & \downarrow f_{R} \\
F_{k}(\vartheta(X)) \rightarrow & \rightarrow & \vartheta\left(X, F_{k}\right) & \stackrel{S}{\rightarrow} & R \otimes F_{k} & \stackrel{J}{\rightarrow} & F_{k}(R)
\end{array}
$$

where the left-hand square is obtained from the isomorphism in Lemma 6.2. It is a simple matter to check that this left-hand square commutes since an equation holds in a function space iff it holds pointwise. As before, the middle square commutes, and since the top and bottom rows are simply the map $\nu_{*}$ induced by the functional calculus $\nu: \theta(X) \rightarrow R$, the outer rectangle commutes by Lemma 3.2. Since $S$ is onto, the right-hand square commutes.

LEMMA 6.4. $R_{S_{k}}=S_{k}(R)$.

Proof. Let $\alpha \in S_{k}(R)$; then $\hat{\alpha}=\gamma_{*}(\alpha) \in S_{k}(C(X))$ by Lemma 3.3 and hence $\operatorname{sp}(\bar{\alpha})=\{\hat{\alpha}(x): x \in X\} \subseteq S_{k}$ by Lemma 6.2. Since $\alpha \in N_{k}(R)$, $f_{R}(\alpha)=0$ and so $\bar{\alpha} \in R_{S_{k}}$ by Lemma 6.3. 
Conversely let $\bar{\alpha} \in R_{S_{k}}$. Note that for each $x \in X,\left(\varepsilon_{x}^{\circ} \circ \gamma\right)_{*}: E_{k}(R) \rightarrow$ $E_{k}$ and that $\left(\varepsilon_{x} \circ \gamma\right)_{*}(\alpha)=\hat{\alpha}(x)$. Since $f_{R}(\alpha)=0$ we have $\hat{\alpha}(x) \in N_{k}$ $(x \in X)$ by Lemma 3.2, and since $\hat{\alpha}(x) \in \operatorname{Sp} \bar{\alpha} \subseteq U$ we in fact have $\hat{\alpha}(x) \in S_{k}$ by Proposition 5.2.

Let $m$ be the maximal ideal of $R$ corresponding to $x$ so that $\varepsilon_{x} \circ \gamma: R \rightarrow R / m$ is just the quotient map. By Lemma 3.3, if $A=$ $\alpha\left(R^{k}\right)$ then $A \otimes_{R} R / m \simeq \hat{\alpha}(x)\left(C^{k}\right) \in \operatorname{Sep}(R / m)$ for every maximal ideal $m$ of $R$. Thus by the local characterisation of separability (Proposition 1.1) $A \in \operatorname{Sep}(R)$ and the lemma follows.

These identifications complete the proof of Theorem 6.1 since the map induced by $\gamma$ on $R_{S_{k}}$ is clearly $\gamma_{*}$ on $S_{k}(R)$ and the topologies induced on these two sets come from equivalent norms on $R^{n}$.

THEOREM 6.5. The map $A \leadsto A \otimes_{R} C(X)$ is a semigroup isomorphism from $\operatorname{Sep}(A)$ to $\operatorname{Sep} C(X)$.

Proof. As described in $§ 1$, the map is certainly a semigroup homomorphism.

Let $B \in \operatorname{Sep} C(X)$ and choose $k>0$ and $\beta \in S_{k}(C(X))$ with $\beta\left(C(X)^{k}\right) \simeq B$. From Theorem 6.1 there is some $\alpha \in S_{k}(R)$ with $\hat{\alpha} \sim \beta$, whence $\hat{\alpha}(C(X))^{k} \simeq B$ by Proposition 4.2. But $\hat{\alpha}=\gamma_{*}(\alpha)$ and so if $A=\alpha\left(R^{k}\right)$ we have $A \otimes_{R} C(X) \simeq \gamma_{*}(\alpha)(C(X))^{k} \simeq B$ using Lemma 3.3. Thus the map is surjective.

To check injectivity let $A_{0}, A_{1} \in \operatorname{Sep}(R)$ with $A_{0} \otimes_{R} C(X) \simeq$ $A_{1} \otimes_{R} C(X)$. Choose $k>0$ and $\alpha_{i} \in S_{k}(R)$ such that $\alpha_{i}\left(R^{k}\right) \simeq A_{i}(i=$ $0,1)$. Then by hypothesis $\hat{\alpha}_{0}\left(C(X)^{k}\right) \simeq \hat{\alpha}_{1}(C(X))^{k}$ and so by Proposition 4.2, $\hat{\alpha}_{0} \oplus 0 \sim \hat{\alpha}_{1} \oplus 0$ in $S_{4 k}(C(X))$. By the injectivity part of Theorem 6.1 $\alpha_{0} \oplus 0 \sim \alpha_{1} \oplus 0$ and hence $A_{0} \simeq A_{1}$. Then

Corollary 6.6. Let $A \in \operatorname{Sep}(R)$ and let $B=A \otimes_{R} C(X) \in \operatorname{Sep} C(X)$.

(i) $A$ is faithful iff $B$ is

(ii) $A$ is commutative iff $B$ is

(iii) $A$ is central iff $B$ is.

Proof. It is elementary that if $A$ has one of the above properties, then so does $B$. 
Note that in general we can write $R=\operatorname{tr}(A) \oplus \operatorname{ann}(A)$, the direct sum of the trace and annihilator ideals [6, I.1.9] and hence obtain an idempotent $e \in R$ with $e R=\operatorname{ann}(A)$. Working in $B=A \otimes_{R} C(X)$ we have

$$
(1 \otimes 1) \hat{e}=1 \otimes \hat{e}=e \cdot 1 \otimes 1=0 \quad \text { since } e \in \operatorname{ann}(A) .
$$

Thus if $B$ is faithful, $\hat{e}$ is the zero idempotent in $C(X)$, and hence by the Silov idempotent theorem $e=0$. Thus $\operatorname{ann}(A)=(0)$ and $A$ is faithful.

Let $e \in A \otimes A^{0}$ be the separability idempotent for $A$. Then $Z(A)$, the centre of $A$ is simply $e \cdot A$; from this it is clear that $Z(B)=Z(A) \otimes_{R} C(X)$. By [6, II.3.8] we know that $Z(A)$ is itself a separable algebra, and since $e$ is idempotent in $A \otimes A^{0}$, the projection $a \leadsto$ a.e. shows that $Z(A)$ is also projective.

Now assume that $B$ is commutative. Then $Z(A) \otimes_{R} C(X) \simeq$ $A \otimes_{R} C(X)$ and so by the injectivity part of Theorem 6.5, $A$ and $Z(A)$ are isomorphic $R$-algebras and thus $A$ is commutative.

If instead we assume that $B$ is central we have $Z(A) \otimes_{R} C(X) \simeq$ $C(X) \simeq R \otimes_{R} C(X)$ so $Z(A) \simeq R$ as $R$-algebras, again by Theorem 6.5. Thus $A$ is central.

This corollary enables us to specialise the isomorphism of Theorem 6.5 both to $\operatorname{Cov}(R)$, the subsemigroup of $\operatorname{Sep}(R)$ consisting of those commutative algebras which are faithfully projective as modules, and to $\mathrm{Az}(R)$, the subsemigroup of $\operatorname{Sep}(R)$ consisting of the central $R$-algebras. phisms

COROllary 6.7. The map $A \leadsto A \otimes_{R} C(X)$ induces semigroup isomor-

$$
\begin{aligned}
\operatorname{Cov}(R) & \rightarrow \operatorname{Cov} C(X) \\
\operatorname{Az}(R) & \rightarrow \operatorname{Az} C(X)
\end{aligned}
$$

and hence a group isomorphism

$$
\operatorname{Br}(R) \rightarrow \operatorname{Br} C(X) .
$$

\section{REFERENCES}

1. N. Bourbaki, Théories Spectrales, Hermann, Paris 1967.

2. L. N. Childs, On covering spaces and Galois extensions, Pacific J. Math., 37 (1971), 29-33.

3. I. Craw, A condition equivalent to the continuity of characters on a Fréchet algebra, Proc. London Math. Soc., 23 (1971), 31-52.

4. , Galois extensions of a Banach algebra, J. Funct. Anal.,27 (1978), 170-178. 
5. I. Craw, I. Raeburn and J. L. Taylor, Automorphisms of Azumaya algebras over a commutative Banach algebra, submitted for publication.

6. F. DeMeyer and E. Ingraham, Separable algebras over commutative rings, Lecture Notes in Math. 181, Springer-Verlag, New York, 1971.

7. J. Dieudonné, Treatise on Analysis III, Academic Press, New York, 1972.

8. O. Forster, Function theoretische Hilfsmittel in der theorie der kommutativen Banach-algebren, Jber. Deutsch. Math.-Verein., 76 (1974), 1-17.

9. H. Grauert, Analytische Faserungen uber holomorphvollstandigen Raumen, Math. Ann., 135 (1958), 263-273.

10. A. Grothendieck, Le groups de Brauer I: algèbres d'Azumaya et interprétations deverses, Séminaire Bourbaki, 1964/65 Exposé 290.

11. B. Johnson, Perturbations of Banach algebras, Proc. London Math. Soc., 34 (1977), 439-458.

12. A. Magid, Algebraically separable extensions of Banach algebras, Michigan Math. J., 21 (1974), 137-143.

13. E. Novodvorskii, Certain homotopical invariants of spaces of maximal ideals, Mat. Z., 1 (1967), 487-494.

14. M. Orzech and C. Small, The Brauer Group of Commutative Rings, Marcel Dekker, New York, 1975.

15. I. Raeburn, The relationship between a commutative Banach algebra and its maximal ideal space, J. Funct. Anal., 25 (1977), 366-390.

16. I. Raeburn and J. L. Taylor, Hochschild cohomology and perturbations of Banach algebras, J. Funct. Anal., 25 (1977), 258-266.

17. K. J. Ramspott, Stetige und holomorphe Schnitte in Bündeln mit homogener Faser, Math. Z., 89 (1965), 234-246.

18. J. L. Taylor, Topological invariants of the maximal ideal space of a Banach algebra, Advances in Math., 19 (1976), 149-206.

19. Twisted products of Banach algebras and third Cech cohomology, in Lecture Notes in Math., Vol. 575, Springer-Verlag, New York, 1977.

20. A bigger Brauer group, preprint.

21. W. Zame, Covering spaces and the Galois theory of commutative Banach algebras, preprint.

Received March 29, 1981.

UNIVERSITY OF ABERDEEN

ABERDEEN, SCOTLAND

AND

MACKIE ACADEMY

STONEHAVEN, ScotLAND 


\title{
PACIFIC JOURNAL OF MATHEMATICS EDITORS
}

\author{
Donald BABBITT (Managing Editor) \\ University of California \\ Los Angeles, CA 90024 \\ Hugo Rossi \\ University of Utah \\ Salt Lake City, UT 84112 \\ C. C. Moore and Arthur Ogus \\ University of California \\ Berkeley, CA 94720
}

\author{
J. Dugundi \\ Department of Mathematics \\ University of Southern California \\ Los Angeles, CA 90089-1113
}

R. FinN and H. SAMELSON

Stanford University

Stanford, CA 94305

\section{ASSOCIATE EDITORS}
R. ARENS
E. F. BECKENBACH
B. H. NeumanN
F. WOLF
K. YoSHIDA (1906-1982)

\section{SUPPORTING INSTITUTIONS}

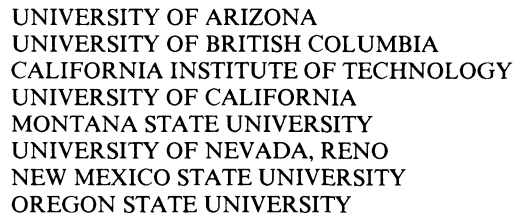

UNIVERSITY OF ARIZONA

UNIVERSITY OF BRITISH COLUMBIA

CALIFORNIA INSTITUTE OF TECHNOLOGY

UNIVERSITY OF CALIFORNIA

MONTANA STATE UNIVERSITY

UNIVERSITY OF NEVADA, RENO

NEW MEXICO STATE UNIVERSITY

OREGON STATE UNIVERSITY

\author{
UNIVERSITY OF OREGON \\ UNIVERSITY OF SOUTHERN CALIFORNIA \\ STANFORD UNIVERSITY \\ UNIVERSITY OF HAWAII \\ UNIVERSITY OF TOKYO \\ UNIVERSITY OF UTAH \\ WASHINGTON STATE UNIVERSITY \\ UNIVERSITY OF WASHINGTON
}

The Supporting Institutions listed above contribute to the cost of publication of this Journal, but they are not owners or publishers and have no responsibility for its content or policies.

Mathematical papers intended for publication in the Pacific Journal of Mathematics should be in typed form or offset-reproduced (not dittoed), double spaced with large margins. Please do not use built up fractions in the text of the manuscript. However, you may use them in the displayed equations. Underline Greek letters in red, German in green, and script in blue. The first paragraph must be capable of being used separately as a synopsis of the entire paper. In particular it should contain no bibliographic references. Please propose a heading for the odd numbered pages of less than 35 characters. Manuscripts, in triplicate, may be sent to any one of the editors. Please classify according to the scheme of Math. Reviews, Index to Vol. 39. Supply name and address of author to whom proofs should be sent. All other communications should be addressed to the managing editor, or Elaine Barth, University of California, Los Angeles, California 90024.

There are page-charges associated with articles appearing in the Pacific Journal of Mathematics. These charges are expected to be paid by the author's University, Government Agency or Company. If the author or authors do not have access to such Institutional support these charges are waived. Single authors will receive 50 free reprints; joint authors will receive a total of 100 free reprints. Additional copies may be obtained at cost in multiples of 50 .

The Pacific Journal of Mathematics is issued monthly as of January 1966. Regular subscription rate: $\$ 132.00$ a year (6 Vol., 12 issues). Special rate: $\$ 66.00$ a year to individual members of supporting institutions.

Subscriptions, orders for numbers issued in the last three calendar years, and changes of address should be sent to Pacific Journal of Mathematics, P.O. Box 969, Carmel Valley, CA 93924, U.S.A. Old back numbers obtainable from Kraus Periodicals Co., Route 100, Millwood, NY 10546.

The Pacific Journal of Mathematics ISSN 0030-8730 is published monthly by the Pacific Journal of Mathematics at P.O. Box 969, Carmel Valley, CA 93924. Application to mail at Second-class postage rates is pending at Carmel Valley, California, and additional mailing offices. Postmaster: Send address changes to Pacific Journal of Mathematics, P. O. Box 969, Carmel Valley, CA 93924.

PUBLISHED BY PACIFIC JOURNAL OF MATHEMATICS, A NON-PROFIT CORPORATION

Copyright $\odot 1983$ by Pacific Journal of Mathematics 


\section{Pacific Journal of Mathematics}

Vol. 104, No. $2 \quad$ June, 1983

Leo James Alex, Simple groups and a Diophantine equation ........... 257

Herbert James Alexander and John Wermer, On the approximation of

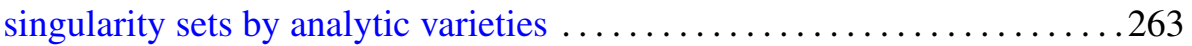

Waleed A. Al-Salam and Mourad Ismail, Orthogonal polynomials associated with the Rogers-Ramanujan continued fraction .......... 269

J. L. Brenner and Roger Conant Lyndon, Permutations and cubic

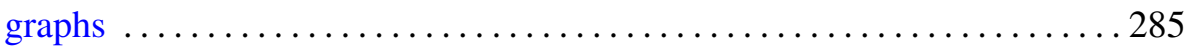

Ian George Craw and Susan Ross, Separable algebras over a commutative

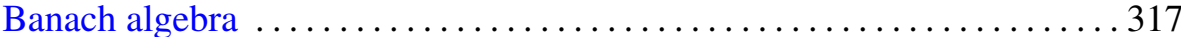

Jesus M. Dominguez, Non-Archimedean Gel'fand theory ............. 337

David Downing and Barry Turett, Some properties of the characteristic of

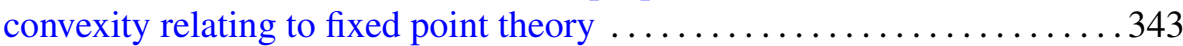

James Arthur Gerhard and Mario Petrich, Word problems for free objects in certain varieties of completely regular semigroups $\ldots \ldots \ldots 351$

Moses Glasner and Mitsuru Nakai, Surjective extension of the reduction

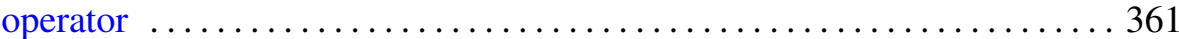

Takesi Isiwata, Ultrafilters and mappings $\ldots \ldots \ldots \ldots \ldots \ldots \ldots \ldots \ldots \ldots \ldots$

Lowell Duane Loveland, Double tangent ball embeddings of curves in $E^{3}$

Douglas C. McMahon and Ta-Sun Wu, Homomorphisms of minimal flows

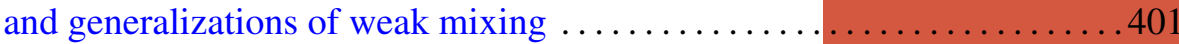

P. H. Maserick, Applications of differentiation of $\mathscr{L}_{p}$-functions to semilattices

Wayne Bruce Powell and Constantine Tsinakis, Free products in the class of abelian $l$-groups

Bruce Reznick, Some inequalities for products of power sums

C. Ray Rosentrater, Compact operators and derivations induced by

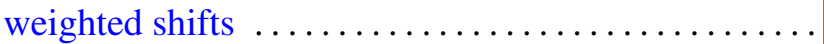

Edward Silverman, Basic calculus of variations

Charles Andrew Swanson, Criteria for oscillatory sublinear Schrödinger equations

David J. Winter, The Jacobson descent theorem 